\title{
La Perspectiva Correlacional en el Estudio de la Relación Investigación-Docencia como Punto de Partida que Permite Conocer los Beneficios de la Práctica Docente de Profesores Destacados
}

\author{
Correlational Perspective in the Study of the Research-Teaching \\ Relationship as a Starting Point that Allows Knowing the Benefits \\ of the Teaching Practice of Outstanding Teachers
}

\author{
Vanessa Cárdenas Novoa * \\ Universidad de Colima, México
}

\section{DESCRIPTORES: \\ Docencia \\ Investigación \\ Educación superior \\ Evaluación de la \\ docencia}

\begin{abstract}
RESUMEN:
Se presentan los resultados de una investigación cuyo objetivo fue conocer la forma en que académicos altamente calificados en investigación y en docencia de una universidad pública mexicana relacionan estas actividades como propuesta de enseñanza dentro del aula y los beneficios que este tipo de docencia trae al aprendizaje de los alumnos. El diseño metodológico tomó como punto de partida el análisis de los resultados de los cuestionarios de opinión de estudiantes para identificar a estos de profesores y se complementó con técnicas de recolección y análisis de datos interpretativas, como la observación en el aula y los grupos focales. Entre los resultados más importantes se encontró que las formas de enseñanza relacionadas con la investigación que estos profesores establecen en sus clases se enfocan más en la trasmisión de los productos de la investigación que en el uso de sus procesos como medio de enseñanza. Se encontró también que las características que distinguen a estos profesores tienen relación directa con el tipo de aprendizajes que desarrollan en sus alumnos. Finalmente, se observó que algunos atributos muy particulares de estos profesores, producto de su quehacer científico, a la vez de representar un beneficio en el aprendizaje de los estudiantes, pueden igualmente limitarlo.
\end{abstract}

\section{KEYWORDS:}

Teaching

Research

Higher education

Teacher evaluation

\section{ABSTRACT:}

This article reports the results of a research on the way of how highly rated scholars link research and teaching as a pedagogical approach and the effects it brings about in the students ' learning. The methodology took as the starting point the results of the students' evaluations to identify this kind of professors and it was complemented by interpretive data collection and analysis techniques like classroom observation and focus groups. The findings include that the links between research and teaching focuses more on the transfer of research products than in the use of its processes as a way of teaching. Likewise, it was found that the distinguishing characteristics of this professors are directly related to the kind of learning outcomes of their students. Finally, it was found that some attributes of these professors, obtained through their scientific activity can act at the same as a benefit for students 'learning or as a barrier.

CÓMO CITAR:

Cárdenas, V. (2021). La perspectiva correlacional en el estudio de la relación investigación-docencia como punto de partida que permite conocer los beneficios de la práctica docente de profesores destacados. Revista Iberoamericana de Evaluación Educativa, 14(1), 135-149. https://doi.org/10.15366/riee2021.14.1.008

*Contacto: vanessa_cardenas@ucol.mx

ISSN: 1989-0397

https://revistas.uam.es/riee 


\section{Introducción}

La relación investigación docencia en la educación superior ha sido un tema de interés y de estudio por varias décadas, debido a que son dos de las actividades con mayor relevancia en el quehacer académico y en la misma misión de la Universidad. Una parte importante de su estudio se ha enfocado en establecer la naturaleza de su relación, la forma en la que se concreta y los beneficios u obstáculos que una obtiene o le representa a la otra.

Para entender esta relación muchos trabajos han optado por el uso de enfoques cuantitativos a través de una perspectiva correlacional, en la que ambas tareas son tomadas como actividades separadas, convertidas en medidas objetivas de productos, la docencia en términos de las calificaciones obtenidas por medio de la evaluación que los estudiantes realizan de la práctica docente de sus profesores y la investigación a través de sus publicaciones. Los resultados de estos trabajos no son concluyentes, si los datos indican que un profesor obtiene altas calificaciones en la evaluación de ambas actividades, se estima que la investigación y la docencia son actividades complementarias, por lo tanto, existe una relación positiva entre ellas. Por el contrario, si los datos indican que el profesor es bien evaluado en una, pero en la otra no, se sugiere la existencia de una relación negativa, ya que estas actividades estarían en conflicto. Hattie y Marsh (1996) proponen un modelo llamado Modelo del Sentido Común, en el que sostienen, derivado de uno de los meta-análisis más completos sobre este objeto de estudio que, estas actividades no se relacionan, que están en conflicto, pues compiten por el tiempo, la energía y el compromiso de los académicos.

En México esta tendencia correlacional hacia el estudio de esta relación puede ser observada, desde la perspectiva negativa, en trabajos como el de De Vries y otros (2008) y Magaña y otros (2007). Estos últimos, por ejemplo, concluyen que "un profesor más habilitado académicamente y más preparado para realizar investigación, descuida considerablemente su comportamiento didáctico y atención a los estudiantes en sacrificio de su labor de investigación, porque obtiene mayor crédito, reconocimiento e incentivos económicos" (Magaña et al., 2007, p. 632). Mientras que, por el lado positivo de la relación se encuentran trabajos como el de García (2003), quien señala que el desempeño o la efectividad docente de los profesores cuya principal área de actividad es la investigación, es mejor que la de los profesores que solo se dedican a la docencia.

Sin embargo, parece insuficiente realizar estudios que se limiten a determinar que existe una relación ya sea positiva o negativa entre estas dos actividades, por lo que se sugiere complementar este tipo de abordajes correlacionales con otros de distinta naturaleza, sobre todo, cuando se trata del estudio de una relación positiva entre la investigación y la docencia, en el sentido de estar en posibilidades de entender y describir buenas prácticas de enseñanza. Se asume que, por la naturaleza de estas aproximaciones cuantitativas, las características más finas de la práctica docente de este tipo de profesores y la forma en la que pudiera estar relacionada con su actividad científica; en términos de observar cómo las herramientas, conocimientos, destrezas y comportamientos que ha obtenido de su quehacer como investigador, se vinculan con su hacer docente e impactan en el aprendizaje de sus estudiantes; no pueden ser observadas ni descritas a detalle. Se reconoce que las calificaciones de los cuestionarios de evaluación de los estudiantes sobre la efectividad de la enseñanza pueden ser utilizadas para ayudar a identificar a profesores y tipos de instrucción ejemplares (Felman, 2007), pero también se reconoce que no cubren todas las manifestaciones de una actividad tan compleja como la docencia y, sobre todo, a la fecha, no cubren vertientes en las que la enseñanza se relaciona con la investigación.

Por ejemplo, el instrumento de evaluación de la docencia por parte de los estudiantes utilizado en la Universidad de Colima en México; mismo contexto en el que Magaña y otros (2007) realizaron su estudio; evalúa de forma muy general cinco dimensiones de la práctica docente de los profesores, entre estas: las competencias profesionales, las habilidades y estrategias didácticas, la evaluación del aprendizaje y las actitudes y valores (UCOL, 2012). Lo anterior, probablemente como señala Luna y Torquemada (2008), bajo el supuesto comúnmente aceptado de que estas pocas dimensiones y los reactivos que las representan forman parte de las habilidades genéricas que todo docente debe presentar.

En ese sentido, para entender con más elementos los aspectos positivos de la enseñanza de estos académicos, se sugiere que la opinión de los estudiantes a través de la evaluación que hacen sobre la práctica de sus profesores, sea tomada solo como un indicador de que existe un desarrollo armónico entre estas dos actividades, de que se ha logrado establecer un balance entre ellas; empero, para complementarlo y esta- 
blecer el alcance y las características de la enseñanza de profesores altamente calificados en ambas actividades, se propone que estos cuestionarios sean solo el primer paso de un camino que se complemente con acercamientos más directos y fundamentados teóricamente en marcos que expliquen este enfoque específico de enseñanza.

En concordancia con lo anterior, la investigación que se presenta se centró en determinar la forma en la que seis profesores investigadores de la Universidad de Colima en México; reconocidos como profesores destacados por sus alumnos a través de los cuestionarios de opinión, y con una alta y distinguida actividad en la investigación; relacionan la investigación y la docencia como forma de enseñanza dentro del aula, para posteriormente conocer, desde la misma voz de los estudiantes, los beneficios y el tipo específico de aprendizajes que obtienen de una docencia bien evaluada y que además se vincula con la investigación.

\section{Fundamentación Teórica}

Entre los aportes teóricos que describen este objeto de estudio, se encuentra una vertiente que estudia la direccionalidad de los beneficios de la relación investigación docencia, es decir, cuál de las dos actividades se ve más favorecida con su integración. Los trabajos que se han ocupado de este tema (Alvarado y Flores, 2010; Deem y Lucas, 2005; Elton, 2001; Grant y Wakelin, 2009; Prieto y Fernández, 2012), establecen que la mayoría del tiempo los beneficios de esta integración se dirigen hacia una sola actividad, regularmente la docencia es la más favorecida y, sucede precisamente, cuando la investigación funge como un enfoque pedagógico cuyo principal objetivo es el aprendizaje del estudiante.

Existen distintos modelos que explican el funcionamiento de este tipo de docencia, entre los más importantes se encuentra el de Jenkins y Healey (2005) que representa un esfuerzo significativo en el avance y entendimiento de esta relación como forma de enseñanza.

\subsection{Modelo de Healey y Jenkins}

Este modelo fue propuesto originalmente por Griffiths (2004) en su afán de ampliar el conocimiento sobre la frase "vincular la docencia con la investigación". La clasificación que este autor realizó, la hizo con base en los distintos usos que se le da a la investigación como herramienta pedagógica para mejorar la docencia, resultando en las siguientes cuatro propuestas de enseñanza:

- Docencia dirigida por la investigación: En este tipo de docencia el currículo se estructura con base en los contenidos del curso. Se enfatiza en la comprensión de los productos de la investigación, ya sean del propio docente o de otros investigadores, pero no se presta atención a los procesos de la investigación. En sí, la vinculación sucede cuando se aprende un conjunto de resultados provenientes de distintos trabajos de investigación.

- Docencia orientada hacia la investigación: Ocurre cuando el currículo enfatiza en los procesos por los cuales el conocimiento es generado. Los estudiantes aprenden los procesos de investigación y sus métodos. Se presta especial atención a la enseñanza de habilidades de investigación.

- Docencia basada en la investigación: En la docencia basada en la investigación los estudiantes aprenden los contenidos del curso como investigadores. El profesor se enfoca en que los estudiantes aprendan los procesos de la investigación a través de métodos de enseñanza como el Aprendizaje Basado en Problemas, en proyectos o estudios de caso.

- Docencia informada por la investigación: Se trata de un tipo de enseñanza informada por la investigación pedagógica. Sucede cuando el profesor ha realizado investigación sobre el proceso de enseñanza aprendizaje de manera sistemática, para favorecer la experiencia de aprendizaje de sus estudiantes.

Sin embargo, Healey (2005) sugiere eliminar de esta clasificación esta última propuesta, la Docencia Informada por la Investigación, argumentando que en esa vinculación la investigación y la docencia no se 
integran para beneficiar de forma directa el aprendizaje de los estudiantes, sino la práctica docente del profesor. Se trata, como argumenta Cervantes (2019), de entender la investigación desde su papel en los procesos de formación docente, a través de un camino de autodescubrimiento que lleva a los profesores a la reconfiguración de creencias y a la transformación de su práctica docente. Por tal motivo, Healey (2005) propone sustituirla por la Docencia Asesorada por la Investigación, en la que el currículo enfatiza un aprendizaje enfocado en la discusión y escritura de ensayos y artículos de investigación y en la que el estudiante aprende a partir de las discusiones actuales de la disciplina.

En ese mismo tenor de perfeccionar el modelo, Jenkins y Healey (2005) proponen explicarlo a través de un cuadrante con dos dimensiones o ejes. El eje horizontal revela, si el profesor vincula su enseñanza con la investigación tomando de ésta sus productos o sus procesos y problemas; mientras que, el eje vertical se enfoca en el papel de los estudiantes en cada propuesta de enseñanza, pudiendo actuar como participantes o como audiencia de estos procesos, para de esa forma, diferenciar las propuestas pedagógicas centradas en el estudiante de las centradas en el profesor.

La explicación gráfica del modelo se muestra en la Figura 1.

\section{Figura 1}

Modelo de Healey y Jenkins

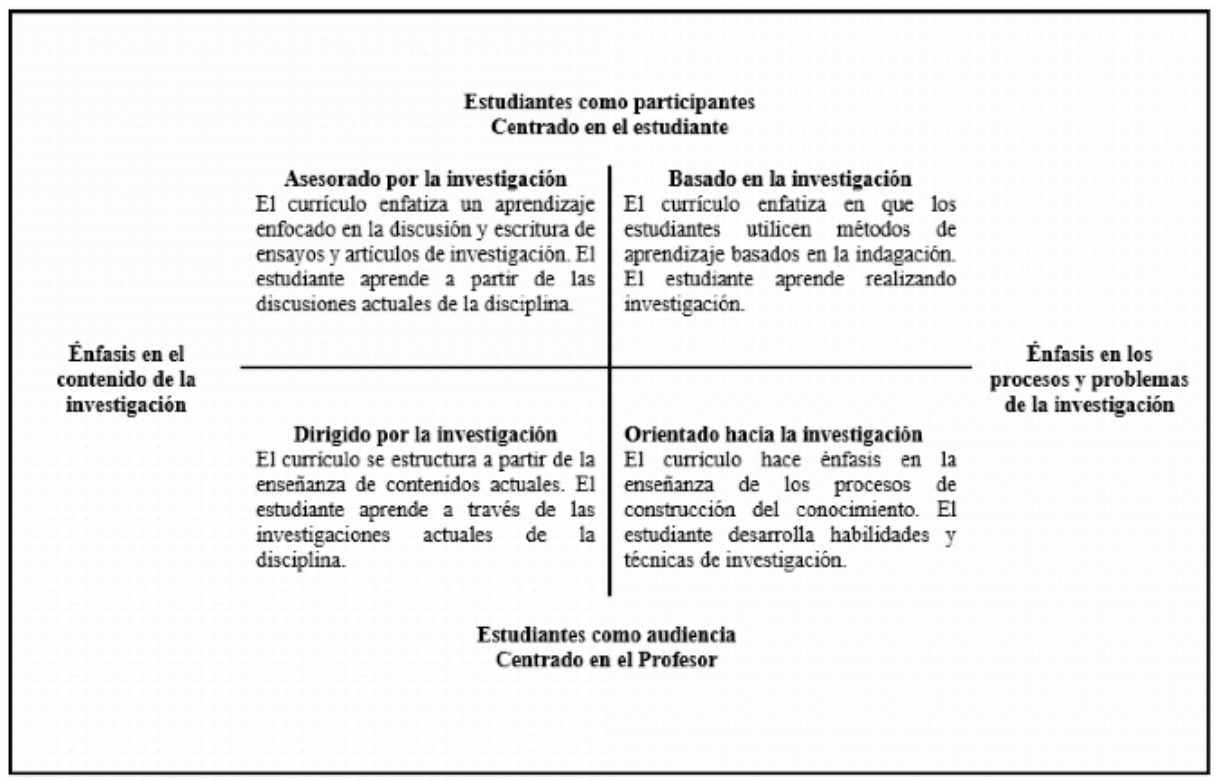

Nota. Jenkins y Healey (2005).

\section{Método}

El diseño metodológico realizado para este trabajo forma parte de una investigación más amplia cuyo objetivo principal fue establecer la relación entre las concepciones sobre la enseñanza y el aprendizaje de profesores destacados tanto en la docencia como en la investigación, y las formas en las que vinculan estas actividades en el aula; de igual forma, se recuperaron las opiniones de los estudiantes sobre este tipo de docencia y sobre los beneficios que trae a su aprendizaje. En esa investigación, la aproximación al campo y a los datos se realizaron en tres etapas. El trabajo que ahora se expone, recupera las dos últimas etapas de ese proceso: el acercamiento al aula y a los estudiantes, a través de un enfoque interpretativo apoyado de técnicas de recolección de datos como la observación y los grupos focales.

\subsection{Criterios para la elección de los profesores participantes}

Uno de los elementos principales de esta investigación es el reconocimiento de la utilidad de los resultados de la evaluación docente por parte de los estudiantes como punto de partida que permite precisar con 
más detalle la práctica docente de académicos destacados que se dedican con la misma intensidad tanto a la investigación como a la docencia.

Con lo anterior en mente, la elección de los participantes se basó en cinco criterios; los tres primeros, enfocados en asegurar la participación activa en la investigación de estos profesores, a través de tres programas de evaluación y estímulos nacionales que promueven esta actividad y; los dos últimos, enfocados en cubrir la parte de una práctica docente exitosa:

Criterio 1: Pertenencia al Sistema Nacional de Investigadores, sistema mexicano creado para reconocer la labor de las personas dedicadas a producir conocimiento científico y tecnología.

Criterio 2: Participación en los últimos dos años en el ESDEPED, programa de Estímulos al Desempeño del Personal Docente.

Criterio 3: Contar con el Perfil Deseable otorgado por el PRODEP, programa que distingue a los académicos que cumplen con eficacia y equilibrio sus funciones como la docencia y la investigación.

Criterio 4: Reconocimiento, en cualquiera de los 4 semestres anteriores, gracias a la evaluación de los estudiantes, como profesores con un desempeño docente promedio superior al $85 \%$.

Criterio 5: Mostrar constancia en los resultados de esta evaluación en esos mismos 4 semestres.

En el periodo de tiempo en que se llevó a cabo la investigación, la Universidad de Colima, contaba con 472 Profesores de Tiempo completo, de estos solo 34 ingresaron a los tres programas de evaluación y estímulos ya mencionados y estuvieron frente a grupo en los últimos 4 semestres, lo que indicaría que también fueron evaluados por sus estudiantes durante ese lapso de tiempo. De estos 34 profesores solo 21 obtuvieron una evaluación promedio mayor al $85 \%$. Los datos de estos 21 profesores se muestran en el Cuadro 1.:

Cuadro 1

Profesores Investigadores de la Universidad de Colima que cumplían con los criterios de selección

\begin{tabular}{|c|c|c|c|c|c|c|c|c|c|c|c|c|}
\hline & $\begin{array}{l}\text { Prof } \\
\text { H/M } \\
\end{array}$ & $\begin{array}{l}\text { ES } \\
16 \\
\end{array}$ & $\begin{array}{l}\text { ES } \\
15 \\
\end{array}$ & SNI & $16 / 2$ & $16 / 1$ & $15 / 2$ & $15 / 1$ & Prom. & Alum & Facul & $\begin{array}{c}\text { Área } \\
\text { SNI }\end{array}$ \\
\hline 1 & M & 6 & 7 & 1 & $94,4 / 11$ & $97,1 / 32$ & $94,4 / 29$ & $97,0 / 24$ & 95,7 & 96 & Psicología & IV \\
\hline 2 & M & 7 & 7 & 1 & $94,3 / 70$ & $96 / 84$ & $94,3 / 61$ & $96,6 / 108$ & 95,3 & 323 & Medicina & III \\
\hline 3 & M & 8 & 7 & 1 & $93,5 / 46$ & $95,6 / 27$ & $97,03 / 39$ & $93,9 / 41$ & 95 & 153 & Telemática & IV \\
\hline 4 & M & 5 & 6 & 1 & $94,6 / 67$ & $94,8 / 53$ & $92,9 / 48$ & $95,58 / 40$ & 94,4 & 208 & $\begin{array}{l}\text { Mercadotec- } \\
\text { nia }\end{array}$ & $\mathrm{V}$ \\
\hline 5 & M & 6 & 6 & 1 & $91 / 18$ & $91,6 / 16$ & $92,5 / 17$ & $93,41 / 23$ & 92,1 & 74 & $\begin{array}{l}\text { Ciencias /C. } \\
\text { Químicas }\end{array}$ & $\mathrm{I} / \mathrm{II}$ \\
\hline 6 & $\mathrm{H}$ & 7 & 7 & 1 & $91,8 / 62$ & $90,8 / 92$ & $92,6 / 67$ & $89,6 / 51$ & 91,2 & 272 & Economía & $\mathrm{V}$ \\
\hline 7 & $\mathrm{H}$ & 5 & 5 & 1 & $96,5 / 23$ & $87,7 / 64$ & $90,7 / 74$ & $86,8 / 39$ & 90,4 & 200 & $\begin{array}{l}\text { Biológicas y } \\
\text { Agrop / } \\
\text { Químicas }\end{array}$ & II \\
\hline 8 & $\mathrm{H}$ & 7 & 6 & 1 & $92,7 / 65$ & $92,7 / 68$ & $89,8 / 56$ & $85,2 / 43$ & 90,1 & 232 & $\begin{array}{l}\text { Medicina } \\
\text { Veterinaria }\end{array}$ & VI \\
\hline 9 & $\mathrm{H}$ & 7 & 7 & 1 & $91,7 / 59$ & $92,3 / 53$ & $89,4 / 61$ & $87,2 / 55$ & 90,1 & 228 & Medicina & III \\
\hline 10 & M & 7 & 5 & 1 & $85,0 / 51$ & $92,7 / 55$ & $88,1 / 58$ & $93,8 / 53$ & 89,9 & 217 & $\begin{array}{l}\text { Letras y Co- } \\
\text { municación }\end{array}$ & IV \\
\hline 11 & M & 6 & 7 & 1 & $87,4 / 47$ & $88,6 / 14$ & $88,3 / 23$ & $95,24 / 6$ & 89,8 & 90 & $\begin{array}{l}\text { Ciencias Ma- } \\
\text { rinas }\end{array}$ & I \\
\hline 12 & $\mathrm{H}$ & 7 & 6 & 1 & $89,2 / 86$ & $86,7 / 62$ & $91 / 108$ & $90,1 / 52$ & 89,2 & 308 & $\begin{array}{l}\text { Contabilidad } \\
\text { y Admon }\end{array}$ & $\mathrm{V}$ \\
\hline 13 & M & 5 & 6 & 1 & $87,6 / 7$ & $90 / 15$ & $86,1 / 33$ & $91,8 / 31$ & 88,8 & 86 & $\begin{array}{l}\text { Ciencias Ma- } \\
\text { rinas }\end{array}$ & I \\
\hline
\end{tabular}




\begin{tabular}{|c|c|c|c|c|c|c|c|c|c|c|c|c|}
\hline 14 & $\mathrm{M}$ & 7 & 8 & 2 & $87,2 / 54$ & $91,1 / 38$ & $93,55 / 17$ & $82,4 / 43$ & 88,5 & 152 & $\begin{array}{l}\text { Medicina / } \\
\text { Biológicas y } \\
\text { Agrop }\end{array}$ & II/III \\
\hline 15 & $\mathrm{H}$ & 8 & 6 & 1 & $84,9 / 88$ & $86,6 / 102$ & $93 / 101$ & $89,7 / 77$ & 88,5 & 368 & $\begin{array}{l}\text { Conta y Ad- } \\
\text { mon } \\
\text { Mercado }\end{array}$ & V \\
\hline 16 & $\mathrm{H}$ & 6 & 7 & 2 & $81,8 / 31$ & $94,37 / 17$ & $87,3 / 28$ & $87,6 / 24$ & 87,7 & 100 & Ciencias & I \\
\hline 17 & $\mathrm{H}$ & 6 & 7 & 1 & $90,7 / 40$ & $88,6 / 17$ & $86,3 / 45$ & $84,5 / 61$ & 87,5 & 163 & FIME & VII \\
\hline 18 & $\mathrm{H}$ & 8 & 7 & 1 & $90,7 / 73$ & $83,3 / 72$ & $86,1 / 72$ & $87,2 / 103$ & 86,8 & 320 & Medicina & III \\
\hline 19 & $\mathrm{M}$ & 8 & 6 & 1 & $70,5 / 22$ & $95,64 / 23$ & $88,8 / 53$ & $88,4 / 44$ & 85,8 & 142 & $\begin{array}{l}\text { Arquitectura y } \\
\text { Diseño }\end{array}$ & IV \\
\hline 20 & M & 8 & 5 & 1 & $81,3 / 34$ & $90 / 39$ & $78,1 / 33$ & $92,4 / 43$ & 85,45 & 149 & $\begin{array}{l}\text { Arquitectura y } \\
\text { Diseño }\end{array}$ & IV \\
\hline 21 & $\mathrm{M}$ & 7 & 7 & 1 & $93,8 / 13$ & $86,8 / 11$ & $76,6 / 4$ & $84,7 / 10$ & 85,4 & 38 & Medicina & III \\
\hline
\end{tabular}

Nota.H/M:Hombre/Mujer; ES16:EDEPED 2016;ES15:ESDEPED 2015;16-2: segundo semestre del año 2016/16-1: primer semestre del año 2016; 15-2: segundo semestre del año 2015 / 15-1: primer semestre del año 2015; Alum: total de alumnos que los evaluaron en ese periodo de tiempo. Elaboración propia a partir de UCOL (2015).

Ahora bien, debido a que esta investigación se construyó pensando en un estudio a profundidad, a través de técnicas de recolección de datos que requieren para su ejecución y análisis un tiempo considerable, como es el caso de la observación directa dentro del aula y los grupos focales; se optó por incluir un número reducido de profesores y, en consecuencia, de alumnos.

Así pues, la reducción de este número inicial de profesores se basó, primero, en el promedio de su desempeño y también en su disponibilidad para participar en el estudio; de igual forma, se cuidó que hombres y mujeres quedaran igualmente representados. El resultado final de este proceso de selección se muestra en el Cuadro 2, donde también se presentan los seudónimos elegidos de manera arbitraria que se utilizaron para referirse a cada uno de los 6 profesores participantes.

Cuadro 2

Datos de los Profesores Investigadores que Participaron en el Estudio

\begin{tabular}{|c|c|c|c|c|c|c|c|c|c|c|c|c|}
\hline & $\begin{array}{l}\text { Prof } \\
\text { H/M }\end{array}$ & $\begin{array}{c}\text { ES } \\
16 \\
\end{array}$ & $\begin{array}{c}\text { ES } \\
15 \\
\end{array}$ & SNI & $16 / 2$ & $16 / 1$ & $15 / 2$ & $15 / 1$ & Prom. & Alum & Facul & $\begin{array}{c}\text { Área } \\
\text { SNI }\end{array}$ \\
\hline CARY & M & 7 & 7 & 1 & $94,3 / 70$ & $96 / 84$ & $94,3 / 61$ & $96,6 / 108$ & 95,3 & 323 & Nutrición & III \\
\hline MARA & M & 8 & 7 & 1 & $93,5 / 46$ & $95,6 / 27$ & $97,03 / 39$ & $93,9 / 41$ & 95 & 153 & Telemática & IV \\
\hline MADY & M & 5 & 6 & 1 & $94,6 / 67$ & $94,8 / 53$ & $92,9 / 48$ & $95,58 / 40$ & 94,4 & 208 & $\begin{array}{l}\text { Mercado- } \\
\text { tecnia }\end{array}$ & V \\
\hline OSIEL & $\mathrm{H}$ & 5 & 5 & 1 & $96,5 / 23$ & $87,7 / 64$ & $90,7 / 74$ & $86,8 / 39$ & 90,4 & 200 & $\begin{array}{l}\text { Biológicas } \\
\text { y Agrop / } \\
\text { Químicas }\end{array}$ & II \\
\hline GABO & $\mathrm{H}$ & 7 & 6 & 1 & $92,7 / 65$ & $92,7 / 68$ & $89,8 / 56$ & $85,2 / 43$ & 90,1 & 232 & $\begin{array}{l}\text { Medicina } \\
\text { Veterinaria }\end{array}$ & VI \\
\hline NICOLÁS & $\mathrm{H}$ & 6 & 7 & 2 & $81,8 / 31$ & $94,37 / 17$ & $87,3 / 28$ & $87,6 / 24$ & 87,7 & 100 & Ciencias & I \\
\hline $\begin{array}{l}\text { FERNAN- } \\
\text { DO }\end{array}$ & $\mathrm{H}$ & 6 & 7 & 1 & $90,7 / 40$ & $88,6 / 17$ & $86,3 / 45$ & $84,5 / 61$ & 87,5 & 163 & FIME & VII \\
\hline
\end{tabular}

Nota. Elaboración propia.

\subsection{La observación}

La observación que se realizó para conocer las características de estos vínculos, en relación a los medios para sistematizar lo observado, fue de carácter estructurado y se enmarcó en el Modelo de Jenkins y Hea- 
ley (2005). Este tipo de observación permitió establecer con anticipación las categorías de análisis de esta parte de la investigación.

- Categoría 1: descripción de la manera en la que el profesor utiliza la investigación en su clase, con la posibilidad de tomar de ella sus procesos o sus productos.

- Categoría 2: orientación de su enseñanza, pudiendo ser centrada en el alumno o centrada en el profesor. Partiendo de las propiedades de esta combinación, fue posible ubicar dentro de las cuatro formas de enseñanza que propone el modelo de Healey y Jenkins la vinculación investigación docencia que se observó en el aula. En suma, el tratamiento de los datos y su análisis se basó en comparar lo observado con los elementos que distinguen la propuesta teórica ya mencionada.

\subsection{Grupos focales con estudiantes}

La guía de entrevista que se elaboró para este estudio tuvo solo una pregunta disparadora:

¿De qué forma el tipo de enseñanza de sus profesores contribuye a mejorar su aprendizaje? A la par de esta pregunta disparadora también se hicieron algunas preguntas de seguimiento cuando se detectó que los alumnos de forma directa daban su opinión sobre los vínculos entre investigación y docencia que sus maestros implementaron en sus clases y que ya se habían hecho evidentes en la observación.

El tratamiento que se le dio a los datos obtenidos de las ocho entrevistas (los grupos numerosos se dividieron en dos) realizadas a los 116 estudiantes de los seis profesores que participaron en la investigación, consistió en lo que Glaser y Straus (1967, citado en Charmaz, 2006), llaman métodos de comparación constante, en los que a partir de la transcripción de las entrevistas, se extraen unidades de análisis para reducir los datos y facilitar la categorización y codificación, hasta llegar a través de la comparación, a un nivel de codificación axial que permite identificar categorías y subcategorías y especificar sus propiedades y dimensiones (Charmaz, 2006).

\section{Resultados}

De forma general se encontraron tres propuestas distintas en las que estos seis profesores relacionan la investigación y la docencia como forma de enseñanza en el aula. La primera, se da a través de una Docencia Dirigida por la investigación; la segunda, se distingue por ser una Pseudo forma de la Docencia Basada en la Investigación y; la tercera, se caracteriza por el uso simultáneo en el aula de dos propuestas pedagógicas para relacionar estas actividades. Sobre los beneficios de cada una de estas formas de docencia, se encontró que las propuestas de enseñanza que toman los productos de la investigación para realizar el vínculo y en las que el profesor tiene un papel determinante en el proceso de enseñanza aprendizaje, muestran un número menor de beneficios o ventajas para el estudiante, que aquellas en las que estos participan de forma activa a través de los procesos de la investigación.

\subsection{Docencia Dirigida por la Investigación.}

\subsubsection{Nicolás y Gabo}

La relación entre la investigación y la docencia observada en las clases de los profesores Nicolás y Gabo, se da a través de la Docencia Dirigida por la investigación y, por lo tanto, se caracteriza por ser una relación muy básica, en el sentido de tomar de la investigación solo sus productos para alimentar o estructurar el programa de sus asignaturas.

De acuerdo con el modelo de Jenkins y Healey (2005), cuando la relación entre investigación y docencia ocurre de esta forma, la orientación de la enseñanza se centra en el profesor y en la trasmisión de estos productos de la investigación a los estudiantes, lo observado en la clase de estos profesores lo confirma. En concreto, estos dos profesores tienen un enfoque de enseñanza centrado en ellos, de tipo cátedra, en el que el uso de diapositivas se convierte en el medio principal para la trasmisión de esos productos. 


\subsubsection{Beneficios de una Docencia Dirigida por la Investigación}

En cuanto a los beneficios que los estudiantes le encuentran a este tipo de docencia, de forma general, se relacionan con la experiencia laboral del profesor y con la modalidad organizativa de su enseñanza.

Por ejemplo, los estudiantes del profesor Nicolás, señalan que su proceso de aprendizaje se ve beneficiado por la amplia experiencia laboral y científica de su profesor, consideran que debido a que su trabajo como investigador le requiere viajar a distintas partes del mundo, el conocimiento que obtiene y genera de esta actividad se ve reflejado en aula, ya que muchas veces lo comparte con los estudiantes o incluso les muestra en físico los materiales que obtiene en estos viajes.

Estudiante 1, profesor Nicolás: "Que tiene experiencia, en todo el ámbito, él no es geólogo es vulcanólogo, entonces ha visitado muchas partes y pues tiene conocimientos acerca de las rocas que nos trasmite y a veces sus clases son como prácticas o nos trae rocas".

La modalidad organizativa de la enseñanza es otro de los beneficios que los estudiantes del profesor Nicolás mencionan, pues consideran que el enfoque teórico práctico que el profesor adopta, se convierte en una ventaja que les permite aplicar los conocimientos que adquieren en el aula a situaciones reales concretas, en específico, los alumnos mencionan que el conocimiento teórico que se les presenta en clase, lo aplican en las distintas prácticas de campo que realizan durante el semestre.

Estudiante 1, profesor Nicolás: "Las salidas. Porque todo lo que vemos en la clase, nos es más práctico verlo en campo, lo aprendemos mejor, no sé, las características de la piedra y la roca".

Estudiante 3, profesor Nicolás: "Pues igualmente dentro de las salidas, el conocer el lugary saber, porque antes habíamos pasado por ciertos lugares a los que ya fuimos y no pensamos que antes los podíamos ver y sería algo importante pues..."

En el caso del profesor Gabo, sus estudiantes también reconocen que es un profesor con un amplio conocimiento y dominio de su disciplina y con mucha experiencia en su ámbito laboral, consideran que todo el conocimiento que ha acumulado en sus años de práctica, les beneficia en su proceso de aprendizaje, ya que le permite ejemplificar los casos que ven en clases, con las múltiples experiencias que ha tenido en campo.

\section{Estudiante *, profesor Gabo: "Pues que tiene un conocimiento amplio del tema y lo domina bien". \\ Estudiante 1, profesor Gabo: "La experiencia de años”. \\ Estudiante 2, profesor Gabo: "Nos está explicando un tema y saca muchos ejemplos, y que se les puede presentar esto, pero también esto y nos pone un buen".}

Finalmente, estos estudiantes consideran como otro beneficio para su aprendizaje, derivado de la experiencia del profesor Gabo, que los temas de la clase pueden ser ampliados con los temas relacionados con las líneas de investigación o de trabajo del mismo profesor.
Estudiante *, profesor Gabo: "A parte que no solo se ha dedicado a las especies convencionales que nos ofrece la escuela o en la mayoría de las carreras que caballos, vacas, ¿no? También ha trabajado con peces, con fauna acuática, cocodrilos, o sea, aunque nosotros como tal en la carrera no vemos fauna acuática ni vemos la fauna silvestre, él ha tenido más acercamiento, entonces, también tiene esa experiencia a diferentes rangos ¿no? de decir que ha trabajado con esos animales y pues mínimo tiene una idea de lo que te puede decir, él no está especia- lizado en eso, pero pues sabe".

Lo anterior concuerda con los resultados y sugerencias encontrados en otras investigaciones en relación a cómo incorporar la investigación como forma de enseñanza desde el currículo con una orientación en la figura del profesor. Brew (2006), por ejemplo, sugiere que el profesor hable acerca de la investigación que realiza en sus cátedras; Taylor (2007) menciona que se deben crear cursos basados en los intereses de investigación de los profesores, y Healey (2005) sugiere incorporar al currículo los resultados y los datos de las investigaciones de los profesores. Todas estas sugerencias se respaldan en el supuesto de facilitar un puente natural entre las actividades de investigación del profesor y el aula, un puente que en esta investigación ha probado ser de utilidad en el aprendizaje de los alumnos. 


\subsection{Pseudo forma de una Docencia Basada en la Investigación.}

\subsubsection{Profesores Mara y Fernando}

Los vínculos observados en las clases de la profesora Mara y el profesor Fernando, son lo que Lee (2010) encontró y clasificó en su trabajo como pseudoformas de relacionar la investigación y la docencia y suceden cuando desde el currículo se propone el uso de un tipo de docencia relacionado con la investigación, generalmente uno en la que el alumno tiene un rol activo, pero en la práctica sucede lo contrario.

De acuerdo con los estudiantes y con los mismos profesores Mara y Fernando, desde los programas educativos en los que imparten clase se establece un enfoque de enseñanza centrado en el alumno a través de la elaboración de un proyecto integrador, que consiste en un conjunto de actividades articuladas entre sí, que tienen como propósito resolver un problema, se trata pues de aprender investigando y, por lo tanto, se trata de una Docencia Basada en la Investigación. Sin embargo, en la práctica este proyecto de investigación se alimenta de una forma de enseñanza opuesta a sus fundamentos teóricos. A saber, en la metodología de un proyecto integrador se señala que los estudiantes obtienen los saberes necesarios para concretarlo, de cada una de las asignaturas que llevan durante un determinado periodo de tiempo, pero lo observado indica que estos saberes convertidos en los contenidos de esas asignaturas se trasmiten a los estudiantes, no los obtienen ni los construyen de forma activa, son entregados por los profesores a través de sus presentaciones y exposiciones. De esta forma, el propósito de una Docencia Basada en la Investigación se desvirtúa o se diluye, ya que los alumnos obtienen en sus clases, sin necesidad de investigar, las instrucciones o los conocimientos precisos para concretar sus proyectos. Por lo tanto, lo que en realidad implementan estos profesores es una Docencia Dirigida por la Investigación.

\subsubsection{Forma en la que los estudiantes se benefician de una Pseudo forma de la Docencia basada en la Investigación}

Con respecto a los beneficios mencionados por los alumnos de estos profesores, se encontró una opinión muy similar a la de los estudiantes de los profesores Gabo y Nicolás, ya que en ambos casos señalan la experiencia del profesor, el amplio conocimiento que tiene de su disciplina y el enfoque práctico que los profesores adoptan en sus clases, como factores que facilitan su aprendizaje. Esta similitud en sus respuestas pudiera estar relacionada con el hecho de que la erudición del profesor, producto de su actividad como científico y como profesional, juega un papel muy importante y se convierte en un beneficio para el aprendizaje de los alumnos, al reducir de cierta forma las limitaciones de una enseñanza trasmisionista.

Por ejemplo, los alumnos de Mara mencionan que se benefician de la amplia experiencia que tiene esta profesora en su disciplina, pues como en el caso del profesor Gabo, también consideran que gracias al conocimiento que le brinda esta experiencia, su profesora tiene la capacidad de explicar ese conocimiento a través de situaciones reales y no solo dejarlo a un nivel teórico que ellos no logren comprender; señalan que cuando el profesor no tiene esta capacidad, sus explicaciones se quedan en un nivel muy superficial y que se limitan a explicarle al alumno lo que leyeron y no lo que vivieron en términos de una vivencia laboral o científica.

Estudiante 1, profesora Mara: "Pues que tienen como que mayor experienciay te meten más, te exigen, te puedes ir a lo más práctico, que te diga ah mira, esto funciona de esta manera y el otro te da texto, entonces, te explica lo que él leyó".

Sobre el enfoque práctico de la enseñanza como un aspecto que potencia el aprendizaje de los estudiantes, se observó que los alumnos de la profesora Mara confunden lo que es una clase de corte práctico, nuevamente, con su capacidad de explicar los temas a través de ejemplos reales y concretos, basados mayormente en sus experiencias profesionales; ya que en repetidas ocasiones insistieron en que gracias al enfoque práctico que la profesora le da a la clase, ellos aprenden con mayor facilidad. Sin embargo, en las entrevistas y en la observación en el aula, se percibió que los estudiantes no tuvieron la oportunidad de aplicar los conocimientos adquiridos en sus clases a situaciones reales concretas, se trata pues, de beneficiarse de explicaciones en las que el conocimiento se ejemplifique con situaciones cercanas a su realidad.

Estudiante 3, profesora Mara: "Este, bueno, a mí, por ejemplo, yo soy más práctico que teórico, entonces al momento que me diga este tema va a servir para que puedas hacer esto y esto, al momento que te enfrentas a un problema similar ya sabes cómo resolverlo, ya tienes idea qué puedes utilizar". 
En el caso del profesor Fernando, el enfoque práctico de la enseñanza como factor que beneficia el aprendizaje también fue mencionado y relacionado de forma directa y acertada con el proyecto integrador. Los alumnos de este profesor mencionan de forma explícita que el beneficio que le encuentran a este tipo de enseñanza es que los conocimientos que cubren en la clase los ponen en práctica con la elaboración de estos proyectos.

Estudiante 1, profesor Fernando: "Pues es buena (enseñanza basada en la investigación a través del proyecto integrador) porque los temas que vemos los podemos aterrizar en el proyecto".

\subsection{Docencias relacionadas con la Investigación que ocurren de forma simultánea}

\subsubsection{Mady y Cary}

En la tercera forma de relacionar estas actividades como propuestas de enseñanza, se observaron vínculos más elaborados y diversificados, en los que la Enseñanza Basada en la Investigación es el elemento principal que desencadena otros tipos de relaciones entre la investigación y la docencia, en ese sentido, lo encontrado en las clases de las profesoras Mady y Cary abona al entendimiento sobre la pluralidad de formas que puede tener esta relación en un mismo espacio y periodo de tiempo.

En la clase de la profesora Mady se observaron dos vínculos entre la investigación y la docencia con un carácter de interdependencia, pues ambas formas de vincular la docencia con la investigación se necesitan mutuamente para lograr un fin, una vez más, la elaboración grupal de un proyecto integrador que consiste en una investigación de mercado. La clase de la profesora Mady se encarga de que los alumnos se apropien de las técnicas y métodos de investigación necesarios para construir un instrumento que les ayude a recolectar los datos para esa investigación, por tal motivo, utiliza primero una Enseñanza Orientada por la Investigación para desarrollar en los estudiantes esas habilidades y técnicas de investigación y, así, apoyar la Enseñanza Basada en la investigación que se ha planteado desde el currículo con la elaboración del proyecto integrador.

Los vínculos observados en la clase de la profesora Cary, también tienen como común denominador la Enseñanza Basada en la Investigación como el elemento principal que desencadena otros tipos de relaciones entre la investigación y la docencia. La profesora Cary es la única de los seis profesores investigadores del estudio que trabaja con un grupo grande de más de 40 estudiantes. Contrario a lo que se pensaría; por lo que implica trabajar con grupos de esta magnitud en cuestión del tiempo de revisión de trabajos, evaluación y demás actividades; esta profesora se ha alejado de una enseñanza trasmisionista y ha elegido una enseñanza centrada en los alumnos y basada en la investigación, que se caracteriza principalmente porque los estudiantes aprenden los contenidos de la asignatura a través de pequeñas tareas y proyectos de investigación a lo largo del curso, es decir, ha elegido una Docencia Basada en la Investigación. En consecuencia, para asegurar la participación de todos los estudiantes en un grupo así de numeroso, la profesora Cary implementa una estrategia de enseñanza en la que todos los alumnos deben leer y reportar de forma escrita y con anticipación los artículos de investigación de los temas que otros compañeros presentarán en la clase, de esta forma también da pie a una Enseñanza Asesorada por la Investigación, en la que se toman los productos de la investigación para que los alumnos aprendan a través de la discusión, análisis y reporte de sus resultados, pero dentro de un entorno que coloca al estudiante en el centro del proceso de enseñanza aprendizaje.

\subsubsection{Forma en la que los estudiantes se benefician de docencias relacionadas con la Investigación que ocurren de forma simultánea}

Los beneficios que los estudiantes de las profesoras Cary y Mady, le encuentran a este tipo de vínculos entre investigación y docencia son diferentes a las ventajas mencionadas por los alumnos que experimentaron esta vinculación a través de una Docencia Dirigida por la investigación o a través de una Pseudo Docencia Basada en la investigación; en el sentido de que estos aportes se relacionan con el desarrollo de habilidades sociales, de investigación, de pensamiento y de actitudes positivas y de motivación hacia el aprendizaje; y no con características particulares del profesor, como su experiencia o el conocimiento de su disciplina. 
En relación al desarrollo de habilidades sociales como resultado de una Docencia Basada en la Investigación, se encontró que los alumnos de la profesora Mady consideran que gracias a esta forma de enseñanza, con una fuerte inclinación hacia el trabajo en equipo, ellos han desarrollado habilidades de convivencia entre pares, señalan que a través de este tipo de actividades se aprende a identificar y a conocer los gustos y los intereses propios y de otros, para que el trabajo en equipo funcione de la mejor manera.

Estudiante 3, profesora Mady: "Es que como suele ser en equipo este tipo de proyectos, como que también identificas con que personas te gusta relacionarte a ti, con quien te gusta trabajar".

Estudiante 4, profesora Mady: “...la meta importante es sobre las personas con las que te vas relacionando, vas calculando, quienes pueden contribuir contigo, tú tienes que ver cuáles son los equipos de trabajo con los que te vas a ir relacionando...”

Sobre este mismo aspecto, los estudiantes de la profesora Cary mencionan que debido a que en esta forma de enseñanza los resultados de sus trabajos de investigación deben ser expuestos y compartidos con el resto del grupo, tienen que desarrollar habilidades de comunicación, en sus palabras deben "ser creativos", para que sus compañeros entiendan sus explicaciones y se apropien del conocimiento que ellos han investigado.

Estudiante 4, profesora Cary: "También creatividad porque de alguna manera lo tienes que saber explicar a tus compañeros".

Respecto al desarrollo de habilidades de pensamiento como consecuencia de este tipo de docencia, se observó, como lo señala la teoría, que a través de las metodologías de enseñanza basadas en la investigación y fundamentadas en el constructivismo, los estudiantes adquieren

niveles más sofisticados de desarrollo intelectual (Baxter y Magolda, 1999, en Healey 2005). Sobre este punto se encontró que, los estudiantes de la profesora Mady consideran que las tareas que realizan a través del proyecto integrador, los han ayudado a ver de una forma distinta su entorno, mencionan que han aprendido a observar y a analizar para poder comprender los comportamientos y fenómenos que los rodean de una forma más profunda.

Estudiante 2, profesora Mady: "Aprendes a observary a ser más atento de tu entorno".

Estudiante 1, profesora Mady: "Yo creo que se abre el panorama social que tú tienes de lo cotidiano ¿no? por decir, ya no ves las cosas superficialmente, sino que ya identificas más a fondo cada comportamiento que hay, socialmente o individual".

De acuerdo con los estudiantes de la profesora Cary, otro beneficio que han adquirido es el desarrollo de habilidades de investigación, en específico, señalan que han aprendido a leer de forma correcta los datos numéricos o estadísticos que aparecen en los libros o en los distintos reportes de investigación que revisan para realizar sus tareas, comentan que ahora son capaces de visualizar un panorama en el que se incluye una fotografía del fenómeno en distintos planos temporales, es decir, desarrollan la capacidad de visualizar el antes, el ahora y el después del comportamiento de una situación problemática o en estudio.

Estudiante 5, profesora Cary: “... hay veces que en los libros viene las prevalencias y todo eso, y yo antes no les tomaba tanta importancia a las prevalencias, así que de movilidad y todo eso, cómo estamos, cómo estábamos y cómo podríamos estary la Mtra. Cary hace mucho énfasis en esa parte, y hay veces que si pongo atención y digo a no manches si es cierto y antes no le tomaba tanta importancia.

En cuanto a cuestiones relacionadas con el plano psicológico de los estudiantes, en el sentido de involucrar la forma de sentir, de pensar y de comportarse de los alumnos, se encontró que estos tipos de Docencias relacionadas con la investigación desarrollan en el alumno actitudes positivas que sobrepasan el plano académico, así como también se observó que aumentan la curiosidad y el deseo por aprender de los estudiantes.

A saber, los alumnos de la profesora Mady señalan que las tareas y proyectos de investigación que les representan un desafío, los inducen a poner todo su empeño para superarlos, comentan que han aprendido que en el plano académico, el esfuerzo y la perseverancia que le imprimen a la resolución de una tarea, van dirigidos hacia la obtención de una calificación como resultado de su esfuerzo, pero también 
han aprendido que el desarrollo de este tipo de actitudes, les servirá posteriormente para enfrentar los problemas de la vida real con el mismo empeño.

Estudiante 3, profesora Mady: "De las adversidades que puede haber en esto pues, al fin de cuentas es una calificación, pero ya en la vida real vas viendo que no hay calificación... es como ver los empeños".

De la misma forma, se encontró que estas formas de docencia relacionadas con la investigación, pero centradas en el alumno, a la vez de desarrollar en los estudiantes esas actitudes positivas, como se acaba de exponer, también estimulan la curiosidad y como consecuencia el deseo de conocer y de aprender. De acuerdo con los alumnos de ambas profesoras, Mady y Cary, este tipo de enseñanza despierta en ellos la necesidad de entender el porqué de una situación problemática, comentan que la curiosidad por conocer que estimula este tipo de enseñanza los lleva incluso a buscar por cuenta propia más información de la que sus profesoras les piden.

Estudiante 1, profesora Mady: "Bueno en lo personal como que te haces más curioso, como que quieres saber por qué".

Estudiante 2, profesora Cary: "Ir más allá de lo que te deja el profesor, que te nazca la curiosidad de saber más".

Estudiante 6 profesora Cary: "Yo en lo personal siempre me voy con ganas de investigar más o que lo que llevé no fue suficiente, o sea, que siempre tienes que dar como más".

Sobre este aspecto de la curiosidad y el deseo de conocer por cuenta propia, también se encontró que para los estudiantes de la profesora Cary, la forma en la que su profesora lleva sus clases, derivado entre otras cosas de su actividad como investigadora, también los ayuda a aprender por ellos mismo, es decir, opinan que este tipo de enseñanza a la vez de provocar actitudes positivas hacia el aprendizaje y motivarlos a dar lo mejor de sí, también estimula el aprendizaje autónomo.

Estudiante 2, profesora Cary: "También opino que el ser investigador, como ella, sabe investigary sabe que hay muchos, en este caso artículosy, asínos hace, o sea, no nos da la información como tal, hace que nosotros la encontremos por nosotros mismos, y hay otros maestros que nada más pues te dan la información y haz lo que viene en el examen y, pues pasas, pero ella nos hace que investiguemos y ver las diferentes variables que hay en el internet o en los libros".

Los estudiantes de ambas profesoras mencionan como otro de los beneficios de una Docencia Basada en la Investigación, la experiencia de sus profesoras en la investigación. Tal y como lo habían mencionado los alumnos de la profesora Mara y el profesor Gabo, estos estudiantes también consideran que la experiencia que tienen sus profesoras en su disciplina, derivada de sus investigaciones, las llevan a elaborar explicaciones claras en las que ejemplifican o complementan los contenidos teóricos de la clase con sus propias investigaciones.

Estudiante 1, profesora Mady: "Normalmente la información es más relevante y trata de enseñarla con casos reales en base a sus investigaciones y experiencia, es como va completando la teoría de cada uno de los temas".

Estudiante 5, profesora Cary: "Pues a mi en lo personal me gusta mucho, porque a pesar de que vemos lo que marca el programa, este pues aparte de que tú sabes este todo lo teórico, también ella se enfoca a que lo entiendas, o sea, a que tú le puedas dar una utilidad ya que salgas de la carrera o ya que se te presente un problema ya en tu vida profesional, y aparte de que ella aporta cosas que son también relevantes y actuales de lo que ve ella en su investigación".

Estudiante 2, profesora Cary: “...pues comparte su experiencia y creo que es lo más valioso porque ya lleva ella años estudiando y entonces lo comparte con nosotros”.

En suma, sobre este último beneficio se recupera que los estudiantes de todos los profesores del estudio visualizan la forma en la que se implementa la investigación en sus clases como un elemento de actualización del docente en su disciplina, sin embargo, no necesariamente por su aplicación en la resolución de problemas o proyectos en donde ellos intervengan. 


\section{Discusión y conclusiones}

A partir de estos resultados se constata la existencia en esta investigación de las dos aproximaciones que proponen Jenkins y Healey en su modelo para relacionar la investigación y la docencia como forma de enseñanza; así como también que, para llevar a cabo este tipo de docencia, la mayoría de los académicos del estudio se inclinan hacia el uso de los productos de la investigación a través de una enseñanza tradicional. Un asunto un tanto preocupante, en el sentido de que las características y habilidades de estos profesores se pudieran estar desaprovechando con las formas de docencia relacionadas con la investigación que llevan a cabo; ya que de acuerdo con sus estudiantes y con los resultados de otras investigaciones (Esquivel-Martín et al., 2019; Gerda et al., 2012; Healey, 2005; Morán, 2011; Pana et al., 2014; Ozay, 2012) los aprendizajes más profundos y significativos, provienen de propuestas de enseñanza centradas en los alumnos y en los procesos de la investigación.

Esta diferencia, en el tipo de aprendizajes y beneficios que estas distintas propuestas de enseñanza generan, llevan a concluir, primero, que estos profesores si poseen atributos muy particulares, producto de su quehacer científico y, segundo, que estos atributos bien pueden promover docencias basadas en los procesos de la investigación y centradas en los estudiantes o bien pueden obstruirlas.

Entre los atributos de este tipo de profesores que pudieran representar un reto para una docencia centrada en los estudiantes y en los procesos de la investigación, se encuentra la erudición que lo profesores investigadores obtienen como resultado de su quehacer científico y que les permite trasmitir el conocimiento a través de explicaciones magistrales y muy claras, ya que por un lado, como los resultados lo indican, se convierte en un elemento que beneficia de cierta manera; aunque no de la más productiva el aprendizaje de los estudiantes y; más importante aún, se posiciona como una de las características de la práctica docente de este tipo de profesores que los alumnos más valoran; pero, a la inversa, funge también como en un elemento que le impide al profesor avanzar hacia formas de enseñanza relacionadas con la investigación más elaboradas y con mayores beneficios para los estudiantes nuevamente, como posible resultado de la buena aceptación que tiene por parte de los estudiantes y del poco esfuerzo pedagógico que le representa al profesor.

Ahora bien, sobre los atributos que potencia el aprendizaje de los estudiantes, se propone también que existe una relación clara y directa entre estos atributos, habilidades, capacidades o destrezas que distinguen a estos profesores y que obtienen de su práctica en la investigación, con el tipo de aprendizajes, destrezas, habilidades que generan en sus estudiantes. A saber, Prieto (2008), propone que los investigadores poseen ciertas capacidades y destrezas instrumentales, entre estas la capacidad de una muy buena comunicación oral y escrita, la capacidad de análisis y de síntesis, la capacidad organizativa y la disciplina; además de actitudes como el constante deseo de conocer, de aprender, de deconstruir y de construir que no solo se queda en el plano académico, sino que también se convierte en el modo de vida del investigador. En esta investigación se confirmó que todas estas características fueron mencionadas por los estudiantes, como los beneficios que obtienen de la docencia de los profesores que utilizaron en su enseñanza los procesos de la investigación y que colocaron a sus estudiantes en el centro del proceso de enseñanza aprendizaje.

Por lo tanto, se revela un panorama en el que la práctica docente de estos profesores, por un lado, confirma ser acreedora, con justa razón, del reconocimiento de los estudiantes y de la misma institución de ser una docencia destacada y efectiva; pero por otro lado, necesita también aprovechar de mejor manera todas las herramientas que la investigación le otorga a quienes la ejercen de forma activa y dedicada, para cumplir con cabalidad y excelencia su propósito de generar aprendizajes, significativos, profundos y duraderos, como los mencionados en la literatura y como los observados en este estudio. Esta relación entre docencia e investigación indudablemente trae consigo múltiples ventajas en los aprendizajes de los estudiantes, por lo tanto, también se propone que los encargados de la evaluación docente por parte de los estudiantes en las instituciones de educación superior, empiecen a incorporar dentro de los instrumentos diseñados para este fin, los resultados de este tipo de estudios para crear reactivos que revelen la forma en la que los profesores incorporan la investigación en su práctica docente; no solo con fines de selección, promoción o acceso a incentivos complementarios, sino con un enfoque hacia la mejora y hacia el aprendizaje y socialización de buenas prácticas. 


\section{Referencias}

Alvarado, M. y Flores, F. (2010). Percepciones y supuestos sobre la enseñanza de la ciencia. Las concepciones de los investigadores universitarios. Perfiles Educativos, 32(128), 10-26.

Brew, A. (2006). Learning to develop the relationship between research and teaching at an institutional level. New Directions for Teaching and Learning, 107, 13-22. https://doi.org/10.1002/t1.240

Cervantes, E. (2019). Un acercamiento a la formación de docentes como investigadores educativos en México. REICE. Revista Iberoamericana sobre Calidad, Eficacia y Cambio en Educación, 17(4), 59-74. https://doi.org/10.15366/reice2019.17.4.003

Charmaz, K. (2006). Constructing grounding theory. A practical guide through qualitative analysis. Sage.

De Vries, W., González, G., León, P. y Hernández, I. (2008). Políticas públicas y desempeño académico o cómo el tamaño sí importa. Revista de Investigación Educativa, 7, 1-32.

Deem, R. y Lucas, L. (2005). Research and teaching cultures in two contrasting UK policy contexts: Academic life in education departments in five English and Scottish universities. Higher Education, 54(1), 115-133. https://doi.org/10.1007/s10734-006-9010-z

Elton, L. (2001). Research and teaching: Conditions for a positive link. Teaching in Higher Education, 6(1), 43-56. https://doi.org/10.1080/13562510020029590

Esquivel-Martín, T., Bravo-Torija, B. y Pérez Martín, J. M. (2019). Brecha entre investigación y praxis educativas en la enseñanza de Biología. REICE. Revista Iberoamericana sobre Calidad, Eficacia y Cambio en Educación, 17(4), 75-91. https://doi.org/10.15366/reice2019.17.4.004

Felman, K. (2007). Identifying exemplary teachers and teaching: Evidence from student ratings. En R. Peery y J. Smart (Eds.), The scholarship of teaching and learning in higher education: An evidence based perspective (pp. 93143). Springer.

García, B. (2003). La evaluación de la docencia en el nivel universitario: Implicaciones de las investigaciones acerca del pensamiento y la práctica docente. Revista de la Educación Superior, 32(3), 63-70.

Gerda, J., Visser, W., Jan, H., van Driela, R., van der Rijsta, A. y Verlopa, N. (2012). Relating academics' ways of integrating research and teaching to their students' perceptions. Studies in Higher Education, 37(2), 219-234. https://doi.org/10.1080/03075079.2010.536913

Grant, K. y Wakelin, S. (2009). Re-conceptualizing the concept of a nexus? A survey of 12 Scottish IS/IM academics' perceptions of a nexus between teaching, research, scholarship and consultancy. Teaching in Higher Education, 14(2), 133-146. https://doi.org/10.1080/13562510902757146

Griffiths, R. (2004). Knowledge production and the research-teaching nexus: The case of the built environment disciplines. Studies in Higher Education, 29, 709-726. https://doi.org/10.1080/0307507042000287212

Hattie, J. y Marsh, H. (1996). The relationship between research and teaching: A meta-analysis. Review of Educational Research, 66(4), 507-542. https://doi.org/10.2307/1170652

Healey, M. (2005). Linking research and teaching: disciplinary spaces. En R. Barnett (Ed.), Reshaping the university: New relationships between research, scholarship and teaching (pp. 30-42). McGraw-Hill.

Jenkins, A. y Healey, M. (2005). Institutional strategies for linking teaching and research. https://www.heacademy.ac.uk/assets/York/documents/resources/resourcedatabase/id585_institutional_trategies_ to_link_teaching_and_research.pdf

Lee, V. (2010). The power of inquiry as a way of learning. Innovative Higher Education, 36, 149-160. https://doi.org/10.1007/s10755-010-9166-4

Luna, E. y Torquemada, A. (2008). Los cuestionarios de evaluación de la docencia por los alumnos: Balance y perspectivas de su agenda. Revista Electrónica de Investigación Educativa, 10, art 4.

Magaña, M., Montesinos, O. y Hernández, C. (2007). Comparación del nivel de escolaridad para el desempeño docente y académico. Revista Mexicana de Investigación Educativa, 12(33), 615-634. 
Morán, P. (2011). Docencia e investigación en el aula. Una relación imprescindible. IISUE.

Ozay, S. (2012). The dimensions of research in undergraduate learning. Teaching in Higher Education, 17(4), 453-464. https://doi.org/10.1080/13562517.2011.641009

Pana, W., Cottonb, D. y Murrayc, P. (2014). Linking research and teaching: context, conflict and complementarity. Innovations in Education and Teaching International 51(1), 3-14. https://doi.org/10.1080/14703297.2013.847794

Prieto, L. (2008). Características funcionales del investigador establecido. Quórum Académico, 5(2), 135-153.

Prieto, L. y Fernández L. (2012). Investigación-Docencia desde la perspectiva del investigador humanista. Revista de Ciencias Sociales, 28(1), 159-169.

Taylor, J. (2007). The teaching research nexus: A model for institutional management. Higher Education, 54(6), 867884. https://doi.org/10.1007/s10734-006-9029-1

Universidad de Colima (UCOL). (2012). Revisión del proceso de evaluación docente por los estudiantes. UCOL.

\section{Breve CV de la autora}

Profesora por horas de la Facultad de Lenguas Extranjeras de la Universidad de Colima, México. Ha impartido docencia en materias relacionadas con los fundamentos pedagógicos y la formación para la investigación de los profesores de Idiomas. Doctora en Educación por la Universidad Iberoamericana, Ciudad de México. Profesora colaboradora del Cuerpo Académico en consolidación de Lingüística aplicada a la Enseñanza de Lenguas. Actualmente colabora en el comité curricular para el desarrollo de un programa de Maestría en la Facultad de Lenguas Extranjeras. Sus líneas de investigación se enfocan hacia los actores de la investigación, la relación investigación docencia y la cultura y formación académica de los profesores de lenguas. Email: vanessa_cardenas@col.mx

ORCID ID https://orcid.org/0000-0001-8427-2334 